\title{
Optimal Detector for Discrete Transmit Signals in Gaussian Interference Channels
}

\author{
Jungwon Lee \\ Wireless Systems Research \\ Marvell Semiconductor, Inc. \\ 5488 Marvell Ln \\ Santa Clara, CA 95054 \\ Email: jungwon@stanfordalumni.org
}

\author{
Dimitris Toumpakaris \\ Wireless Telecommunications Laboratory \\ Department of Electrical and \\ Computer Engineering \\ University of Patras, Rio, Greece 26500 \\ Email: dtouba@upatras.gr
}

\author{
Wei Yu \\ Department of Electrical and \\ Computer Engineering \\ University of Toronto \\ Toronto, Ontario, M5S 3G4, Canada \\ Email:weiyu@comm.utoronto.ca
}

\begin{abstract}
This paper addresses the design of optimal and nearoptimal detectors for a practical interference channel scenario where the transmitters employ discrete modulation schemes. The conventional detectors, which either ignore the interference or successively detect then cancel the interference, typically assume that the desired signal and/or the interference are Gaussian. This paper proposes detectors that explicitly take into account the modulation formats of both the desired signal and the interference. The optimal maximum-likelihood (ML) detector that minimizes the probability of detection error for a given set of modulation schemes is derived first. A joint minimumdistance detector (MD) is then presented as a low-complexity approximation of the optimal ML detector. It is demonstrated by analysis and by simulation that the proposed detectors can significantly outperform their conventional counterparts. In particular, while the interference-ignorant and the successive interference cancellation detectors are both prone to error floors, the proposed optimal ML and joint MD detectors are not.
\end{abstract}

\section{INTRODUCTION}

The Gaussian interference channel has attracted significant research interest in recent years. The interference channel models many important real-world communication systems, for example, the cellular network where neither base stations nor mobile stations coordinate, or the bundled digital subscriber line (DSL) system with no real-time central-office coordination [1-3]. So, the characterization of the capacity region and the development of practical ways of approaching the capacity of the interference channel are of significant practical interest. From an information theoretic perspective, although a complete characterization of the capacity region of the interference channel is still an open problem (see [4], [5]), significant recent advances have enabled an approximate characterization of the capacity within a constant gap [6]. From a practical perspective, however, the development of interference-aware coding and detection schemes for the interference channel has been largely restricted to either the interference-ignorant or the successive interference-cancellation (SIC) types. Moreover, the design and analysis of conventional detection schemes almost always make the simplifying assumption that both the desired signal and the interference are Gaussian.

This paper makes progress in the design of practical detectors for the interference channel by focusing on the fact that the transmit signals in a practical implementation always belong to discrete signal constellations. Therefore, the design of detectors for the interference channel must explicitly account for the discrete modulation format. Towards this end, this paper proposes the use of maximum-likelihood (ML) detection at the receiver. Unlike the conventional interference cancellation schemes, which rely on the successful detection of the interfering signals, the proposed scheme forms a ML metric on the intended signal directly. By both analytic and simulation means, it is shown that for higher-order modulation formats, the proposed optimal ML detector can significantly outperform the existing conventional detectors for a range of signal-to-interference ratios (SIRs). Further, this paper proposes a low-complexity approximation of the ML detector, called joint minimum-distance (MD) detector, which simplifies implementation with minimal impact on performance.

The idea of joint detection has been studied in the past for the multiple-access channel in the code division multiple access (CDMA) literature [7], where the detector is interested in decoding two independently transmitted signals simultaneously. In this case, joint detection and SIC are both sensible strategies. The setup for the interference channel differs in that only one of the transmitted signals is of interest. Thus, one might expect that treating interference as noise is optimal when the interference is low, while SIC is optimal when the interference is high. The main contribution of this paper is to show that there exists an intermediate range of interference levels where neither ignoring nor completely decoding the interference is optimal. A good strategy in this regime is essentially to detect the desired and the interfering signals jointly, then to discard the detector output for the interference (which may be unreliable anyway, but nevertheless aids the detection of the desired signal). Interestingly, such a joint detector can significantly outperform both the interferenceignorant detector and the SIC detector.

\section{SYSTEM MODEL}

Consider a two-user Gaussian interference channel

$$
\begin{aligned}
& y_{1}[m]=h_{1,1}[m] x_{1}[m]+h_{1,2}[m] x_{2}[m]+z_{1}[m], \\
& y_{2}[m]=h_{2,1}[m] x_{1}[m]+h_{2,2}[m] x_{2}[m]+z_{2}[m],
\end{aligned}
$$


where $x_{j}[m]$ is the transmit signal of user $j$ at time $m$, $h_{i, j}[m]$ is the channel gain from transmitter $j$ to receiver $i$, and $z_{i}[m]$ is the background noise of receiver $i$, for $i=1,2$ and $j=1,2$. The background noise is modeled as a real Gaussian random variable with variance $\bar{\sigma}_{z}^{2}$ for baseband systems and as circularly symmetric complex Gaussian with variance $2 \bar{\sigma}_{z}^{2}$ for passband systems.

The interference channel is traditionally classified as "strong" or "weak" depending on the relative channel gains. This paper, however, is mainly interested in a fading channel scenario in which the transmitters do not know the channel gains, but each receiver $i$ knows both $h_{i, 1}[m]$ and $h_{i, 2}[m]$. In this case, the channel may be strong or weak at different time instances. In addition, the transmitter cannot adapt to the channel, so it must use a fixed finite constellation (e.g. pulseamplitude modulation (PAM), quadrature-amplitude modulation (QAM), or phase shift keying (PSK)). This paper assumes that the modulation formats of both the direct signal and the interference are known to both receivers. This is a reasonable assumption because the modulation information is usually heavily encoded in a preamble. Thus, it is typically possible to decode the modulation information of the interferer even when the regular data are not decodable. Throughout the paper, it is implicitly assumed that transmit symbols are equiprobable. Further, although the time index $m$ was explicitly used to emphasize the variation of the channel gain over time, it will be omitted for simplicity in the rest of the paper.

This paper considers a receiver architecture that performs hard-decision symbol detection followed by decoding. While joint detection and decoding (i.e. soft decoding) can potentially result in better performance, this is quite complicated to do in an interference channel. Moreover, the separation of the detection and the decoding has a merit in that the detection does not depend on the specific coding scheme. For this reason, this paper focuses on the symbol detection problem independent of the channel codes.

\section{Conventional Detectors}

\section{A. Interference-Ignorant Detector}

The interference-ignorant detector performs detection assuming that the interference is a part of the Gaussian noise. The detector for user $i$ simply maps the received symbol to the closest constellation point of the desired transmit signal scaled by the direct channel gain $h_{i, i}$. This detector is optimal when the interference is Gaussian and when it cannot be detected first and then cancelled.

\section{B. Successive Interference Cancellation (SIC) Detector}

The SIC concept has been used on interference channels for the decoding of superposition coded signals [4]. It can also be applied to symbol detection as follows. Receiver 1 obtains an estimate $\hat{x}_{2}$ of $x_{2}$ first, by treating $x_{1}$ as part of Gaussian noise. The receiver then detects $x_{1}$ from $y_{1}-h_{1,2} \hat{x}_{2}$ by mapping $y_{1}-h_{1,2} \hat{x}_{2}$ to the closest constellation point of the desired transmit signal scaled by $h_{1,1}$. Note that the SIC detector does not exploit the knowledge of the modulation scheme of $x_{1}$ when detecting $x_{2}$.

\section{INTERFERENCE-AWARE DETECTORS}

\section{A. Optimal Maximum-Likelihood (ML) Detector}

The derivation of the optimal ML detector is based on the minimization of the probability of detection error $P\left\{\hat{x}_{1} \neq x_{1}\right\}$ for the desired signal $x_{1}$. It is optimal when all constellation points of $x_{1}$ are equiprobable.

By definition, the ML detector is given by

$$
\hat{x}_{1}\left(y_{1}\right)=\underset{x_{1}}{\arg \max } f\left(y_{1} \mid x_{1}\right),
$$

where $f\left(y_{1} \mid x_{1}\right)$ is the conditional pdf of $y_{1}$ given $x_{1}$, which can be represented in terms of the conditional pdf $f\left(y_{1} \mid x_{1}, x_{2}\right)$ :

$$
f\left(y_{1} \mid x_{1}\right)=\sum_{x_{2}} p\left(x_{2}\right) f\left(y_{1} \mid x_{1}, x_{2}\right)=\frac{1}{M_{2}} \sum_{x_{2}} f\left(y_{1} \mid x_{1}, x_{2}\right) \text {, }
$$

where $M_{2}$ is the constellation size of the interferer, and the summation is taken over all $M_{2}$ transmit symbols. The conditional pdf $f\left(y_{1} \mid x_{1}, x_{2}\right)$ of $y_{1}$ given $\left(x_{1}, x_{2}\right)$ is

$$
f\left(y_{1} \mid x_{1}, x_{2}\right)=\frac{1}{\left(\sqrt{2 \pi} \bar{\sigma}_{z}\right)^{l}} \exp \left(-\frac{\left|y_{1}-\sum_{j=1}^{2} h_{1, j} x_{j}\right|^{2}}{2 \bar{\sigma}_{z}^{2}}\right),
$$

where $l=1$ for baseband, and $l=2$ for passband systems. From (2), (3), and (4), the ML detector can be expressed as

$$
\hat{x}_{1}\left(y_{1}\right)=\underset{x_{1}}{\arg \max } \sum_{x_{2}} \exp \left(-\frac{\left|y_{1}-h_{1,1} x_{1}-h_{1,2} x_{2}\right|^{2}}{2 \bar{\sigma}_{z}^{2}}\right) \text {. }
$$

Note that the discrete constellations for $x_{1}$ and $x_{2}$ are explicitly taken into account.

\section{B. Joint Minimum-Distance (MD) Detector}

The optimal ML detector of Section IV-A requires the calculation of a sum of exponential functions. In the following, a low-complexity detector is derived by simplifying the optimal ML detector. When the noise power $\bar{\sigma}_{z}^{2}$ is small, the sum of exponentials can be approximated by the largest exponential:

$$
\begin{aligned}
& \sum_{x_{2}} \exp \left(-\left|y_{1}-h_{1,1} x_{1}-h_{1,2} x_{2}\right|^{2} / 2 \bar{\sigma}_{z}^{2}\right) \\
\approx & \max _{x_{2}} \exp \left(-\left|y_{1}-h_{1,1} x_{1}-h_{1,2} x_{2}\right|^{2} / 2 \bar{\sigma}_{z}^{2}\right) .
\end{aligned}
$$

Then the approximate ML detector can be expressed as

$\hat{x}_{1}\left(y_{1}\right)=\underset{x_{1}}{\arg \max }\left[\max _{x_{2}} \exp \left(-\frac{\left|y_{1}-h_{1,1} x_{1}-h_{1,2} x_{2}\right|^{2}}{2 \bar{\sigma}_{z}^{2}}\right)\right]$.

Noting that an exponential function $\exp (-x)$ is a monotonically decreasing function of $x$, this approximate ML detector can be implemented in two steps. First, estimate $\left(x_{1}, x_{2}\right)$

$$
\left(\widehat{x_{1}, x_{2}}\right)=\arg \min \left|y_{1}-h_{1,1} x_{1}-h_{1,2} x_{2}\right|^{2} \text {. }
$$


As the second step, extract the first element of $\left(\widehat{x_{1}, x_{2}}\right)$ as the estimate, $\hat{x}_{1}$, of $x_{1}$. In the first step, $x_{1}$ and $x_{2}$ are detected jointly based on the minimum distance criterion, where the distance is between the received signal and each combined received constellation point $h_{1,1} x_{1}+h_{1,2} x_{2}$ for all $x_{1}$ and $x_{2}$. Thus, the new detector can be named as the joint minimumdistance (MD) detector.

The first step of the joint MD detector can also be seen to satisfy the optimality criterion of minimizing the probability of error of the transmit signal pair $\left(x_{1}, x_{2}\right)$ when all constellation points of $x_{1}$ and $x_{2}$ are equiprobable, and $x_{1}$ and $x_{2}$ are independent. However, this joint MD detector does not achieve the ultimate goal of minimizing the probability of error for the desired signal $x_{1}$. In the following section, the difference between the optimal ML detector and the joint MD detector is first explained in more detail. Then the advantage of ML and MD detectors over conventional ones is illustrated.

\section{COMPARISON OF THE Detectors}

In this section, the joint MD and optimal ML detectors are compared with the interference-ignorant and SIC detectors in terms of their decision regions, symbol error rates, and complexity. First, the case where both transmit signals are 2-PAM is considered. This simple case serves to highlight the difference between the MD and ML detectors. Then the case of larger constellations is examined, where the MD and ML detectors offer significant advantages over conventional detectors. Via an error floor analysis, it is shown that the MD and ML detectors almost never exhibit error floors over a wide range of SIRs, unlike conventional detectors. Lastly, the complexity of the detectors are compared.

\section{A. 2-PAM Used by Both Transmitters}

Consider the case where 2-PAM is used by both the desired and the interfering transmitters. Then the individual transmit symbols are +1 or -1 , i.e. $\left(x_{1}, x_{2}\right)=( \pm 1, \pm 1)$. Two scenarios are considered depending on whether the desired signal power at the receiver is larger or smaller than the interference power. In a fading channel, in the absence of channel information at the transmitters, both scenarios can occur for a fixed modulation scheme. Fig. 1 shows the received signal constellation and the decision regions. For simplicity, and without loss of generality, it is assumed that the channel gains $h_{1,1}$ and $h_{1,2}$ are positive. The decision regions can also be easily derived when the gains are negative.

The decision regions for Scenario 1, where the interference is weaker than the desired signal, are examined first. From the received signal constellation in Fig. 1(a), it is straightforward to conclude that the decision regions $D_{+1}=\left\{y_{1} \mid y_{1} \geq 0\right\}$ and $D_{-1}=\left\{y_{1} \mid y_{1}<0\right\}$ are the same for the ML, the MD and the interference-ignorant detector.

However, the derivation of the decision regions of the SIC detector is somewhat involved, so it is explained in detail below. The SIC detector first performs detection of the interference:

$$
\hat{x}_{2}= \begin{cases}+1 & \text { if } y_{1} \geq 0 \\ -1 & \text { otherwise }\end{cases}
$$

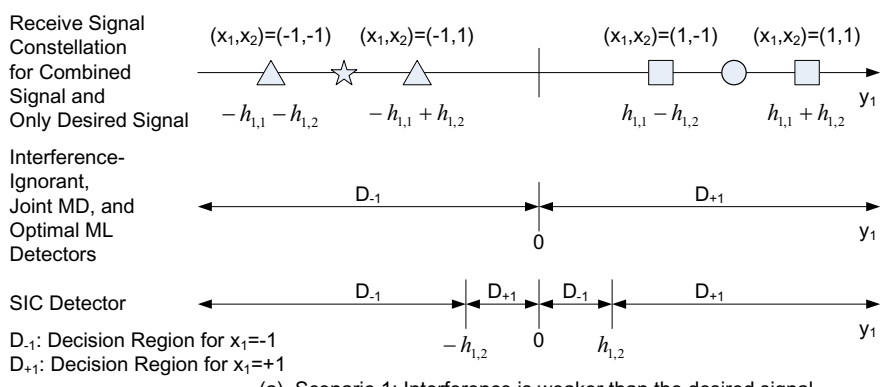

(a) Scenario 1: Interference is weaker than the desired signal.

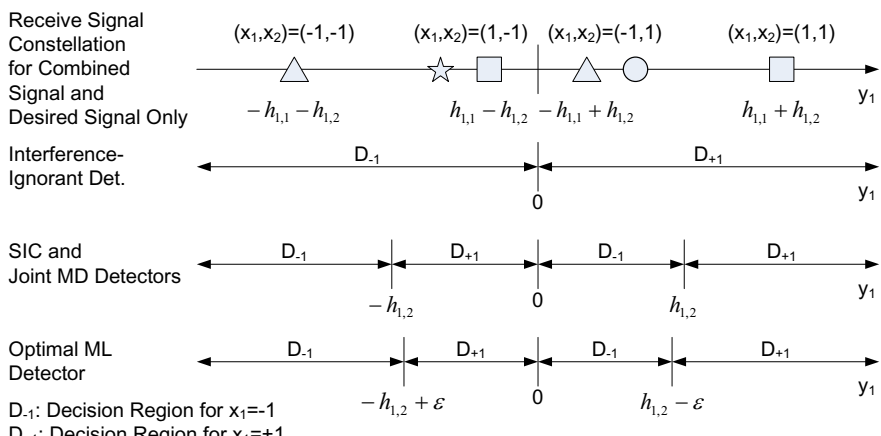

(b) Scenario 2: Interference is stronger than the desired signal.

Fig. 1. Decision regions for Scenarios 1 and 2.

Then the contribution of the interference is subtracted from the received signal based on the estimate, $\hat{x}_{2}$, of the interference.

$$
\tilde{y}_{1}=y_{1}-h_{1,2} \hat{x}_{2} \text {. }
$$

Finally, the decision rule on the desired signal is

$$
\hat{x}_{1}= \begin{cases}+1 & \text { if } \tilde{y}_{1}=y_{1}-h_{1,2} \hat{x}_{2} \geq 0 \\ -1 & \text { otherwise. }\end{cases}
$$

By examining $y_{1} \geq 0$ and $y_{1}<0$ separately, the decision regions can be expressed in terms of the received signal $y_{1}$ as

$$
\begin{aligned}
& D_{+1}=\left\{y_{1} \mid y_{1} \geq h_{1,2}\right\} \cup\left\{y_{1} \mid-h_{1,2} \leq y_{1}<0\right\}, \text { and } \\
& D_{-1}=\left\{y_{1} \mid y_{1}<-h_{1,2}\right\} \cup\left\{y_{1} \mid 0 \leq y_{1}<h_{1,2}\right\} .
\end{aligned}
$$

Next, the decision regions for Scenario 2 in Fig. 1(b), where the interference is stronger than the desired signal, are derived. For the interference-ignorant, the SIC, and the joint MD detector, the decision regions can be derived easily. The decision regions for the optimal ML detector are the most interesting. A closed-form expression does not appear to exist, since the conditional pdf $f\left(y_{1} \mid x_{1}\right)$ is the sum of two exponential functions. In the following, an effort is made to show qualitatively how the decision regions of the optimal ML detector are different from those of the joint MD detector.

The decision regions of the optimal ML detector can be expressed as

$D_{+1}=\left\{y_{1} \mid y_{1} \geq h_{1,2}-\epsilon\right\} \cup\left\{y_{1} \mid-h_{1,2}+\epsilon \leq y_{1}<0\right\}$, and $D_{-1}=\left\{y_{1} \mid y_{1}<-h_{1,2}+\epsilon\right\} \cup\left\{y_{1} \mid 0 \leq y_{1}<h_{1,2}-\epsilon\right\}$,

where $\epsilon$ is a small positive number, which depends on the channel gains $h_{1,1}$ and $h_{1,2}$ as well as the noise variance 
$\bar{\sigma}_{z}^{2}$. In the following, the location of the rightmost decision boundary $y_{1}=h_{1,2}-\epsilon$ is examined. Since, at $y_{1}=h_{1,2}$, $f\left(y_{1} \mid x_{1}=1, x_{2}=1\right)=f\left(y_{1} \mid x_{1}=-1, x_{2}=1\right)$ always, only $f\left(y_{1} \mid x_{1}=1, x_{2}=-1\right)$ needs to be compared with $f\left(y_{1} \mid x_{1}=-1, x_{2}=-1\right)$. The conditional pdfs $f\left(y_{1} \mid x_{1}=\right.$ $\left.1, x_{2}=-1\right)$ and $f\left(y_{1} \mid x_{1}=-1, x_{2}=-1\right)$ have a Gaussian bell shape, and their width is determined by $\bar{\sigma}_{z}^{2}$. The bells are centered at $y_{1}=h_{1,1}-h_{1,2}$ for $f\left(y_{1} \mid x_{1}=1, x_{2}=-1\right)$ and $y_{1}=-h_{1,1}-h_{1,2}$ for $f\left(y_{1} \mid x_{1}=-1, x_{2}=-1\right)$. Thus, the difference between $f\left(y_{1} \mid x_{1}=1, x_{2}=-1\right)$ and $f\left(y_{1} \mid x_{1}=-1, x_{2}=-1\right)$ at $y_{1}=h_{1,2}$ becomes larger as $h_{1,1}$ and ${\overline{\sigma_{z}}}^{2}$ increase, but $h_{1,2}$ decreases. Hence, the optimal ML detector deviates more from the joint MD detector as the signal-to-noise ratio (SNR) decreases, down to when the SIR becomes equal to 1 .

In order to highlight the difference among the detectors, approximate symbol error rate (SER) expressions are presented below. The approximate SER expressions can be obtained using nearest neighbor union bound approach [8]. The derivation of approximate SER expressions can be found in [9] along with the exact SER expressions. The SER of the interferenceignorant detector is

$$
P_{e, I I} \approx \begin{cases}\frac{1}{2} Q(\sqrt{\mathrm{SNR}}-\sqrt{\mathrm{INR}}), & \mathrm{SIR} \geq 1 \\ \frac{1}{2}-\frac{1}{2} Q(\sqrt{\mathrm{INR}}-\sqrt{\mathrm{SNR}}), & \mathrm{SIR}<1,\end{cases}
$$

where $\mathrm{SNR}=\frac{h_{1,1}^{2}}{\bar{\sigma}_{z}^{2}}, \mathrm{INR}=\frac{h_{1,2}^{2}}{\bar{\sigma}_{z}^{2}}, \operatorname{SIR}=\frac{h_{1,1}^{2}}{h_{1,2}^{2}}$, and $Q(x)=$ $\frac{1}{\sqrt{2 \pi}} \int_{x}^{\infty} e^{-t^{2} / 2} d t$ is the Q-function [8]. The SER of the SIC detector is

$$
P_{e, S I C} \approx \begin{cases}\frac{1}{2} Q(\sqrt{\mathrm{SNR}}-2 \sqrt{\mathrm{INR}}), & \mathrm{SIR} \geq 4 \\ \frac{1}{2}-\frac{1}{2} Q(\sqrt{\mathrm{SNR}}-\sqrt{\mathrm{INR}}), & \frac{9}{4} \leq \mathrm{SIR}<4 \\ \frac{1}{2}-\frac{1}{2} Q(2 \sqrt{\mathrm{INR}}-\sqrt{\mathrm{SNR}}), & 1 \leq \mathrm{SIR}<\frac{9}{4} \\ \frac{1}{2} Q(\sqrt{\mathrm{INR}}-\sqrt{\mathrm{SNR}}), & \frac{1}{4} \leq \mathrm{SIR}<1 \\ Q(\sqrt{\mathrm{SNR}}), & \mathrm{SIR}<\frac{1}{4} .\end{cases}
$$

The SER of the joint MD detector is given by

$$
P_{e, M D} \approx \begin{cases}\frac{1}{2} Q(\sqrt{\mathrm{SNR}}-\sqrt{\mathrm{INR}}), & \mathrm{SIR} \geq 1 \\ \frac{1}{2} Q(\sqrt{\mathrm{INR}}-\sqrt{\mathrm{SNR}}), & \frac{1}{4} \leq \mathrm{SIR}<1 \\ Q(\sqrt{\mathrm{SNR}}), & \mathrm{SIR}<\frac{1}{4}\end{cases}
$$

The SER expression for the optimal ML detector is harder to derive, but the SER of the optimal ML detector is upper bounded by the SER of the joint MD detector: $P_{e, M L} \leq$ $P_{e, M D}$.

It is interesting to examine the behavior of the near-optimal joint MD detector for various SIR levels. From (16), it is clear that, for $\mathrm{SIR} \geq 1$, the joint $\mathrm{MD}$ detector behaves as if the minimum distance of the constellation of the desired signal is reduced by the interference. For $\frac{1}{4} \leq \operatorname{SIR}<1$, it behaves as if the minimum distance of the constellation of the interference is reduced by the desired signal. For $\operatorname{SIR}<\frac{1}{4}$, the detection of the desired signal is not affected by the (very strong) interference.

From the SER expressions it can be seen that the joint MD detector reduces to the interference-ignorant detector when the SIR is larger than 1 and to the SIC detector otherwise. Thus, when both transmitters employ 2-PAM, joint detection is equivalent to switching between ignoring the interference and decoding it, depending on the interference strength.

\section{B. Higher-Order Modulation Schemes}

Unlike the 2-PAM case, the joint MD may outperform the ordered SIC significantly when higher-order modulation is employed. This is explained here with an example. Consider the case where 2-PAM is used for the desired signal, but 4PAM is used for the interference, i.e., $x_{1}= \pm 1$ and $x_{2}= \pm \frac{1}{\sqrt{5}}$ or $\pm \frac{3}{\sqrt{5}}$. The detectors are examined using the channel gains $h_{1,1}=4$ and $h_{1,2}=3 \sqrt{5}$ in the absence of background noise. When $\left(x_{1}, x_{2}\right)=\left(-1, \frac{3}{\sqrt{5}}\right)$, the received signal is $y_{1}=h_{1,1} x_{1}+h_{1,2} x_{2}=5$. The interference-ignorant detector will declare $\hat{x}_{1}=1\left(\neq x_{1}\right)$ because $y_{1}>0$. In the case of the SIC detector, the estimate of the interference is $\hat{x}_{2}=\frac{1}{\sqrt{5}}(\neq$ $x_{2}$ ) because the decision boundaries for the interference are located at $y_{1}=0$ and $y_{1}= \pm 3 \sqrt{5} \cdot \frac{1}{2}\left(\frac{1}{\sqrt{5}}+\frac{3}{\sqrt{5}}\right)= \pm 6$, and $0 \leq y_{1}=5<6$. Then the received signal after interference subtraction is $\tilde{y}_{1}=y_{1}-h_{1,2} \hat{x}_{2}=5-3 \sqrt{5} \cdot \frac{1}{\sqrt{5}}=2>0$. Since $\tilde{y}_{1}>0$, the estimate of the desired signal is $\hat{x}_{1}=1\left(\neq x_{1}\right)$. Since both the interference-ignorant detector and the SIC detector fail, the ordered SIC with optimal ordering will also fail. On the other hand, it is clear that the joint MD detector and the optimal ML detector estimate the transmit signal correctly in this case of no noise.

By analyzing the decision regions for any PAM constellation, the performance of the detectors can be examined in the asymptotic case of infinite SNR. This infinite SNR case is of practical interest for systems such as a cellular system with a small cell size, where the system performance is limited by interference rather than by noise. The performance of the detectors in the case of infinite SNR is summarized by the following theorem. A detailed proof based on decision region analysis is given in [9].

Theorem 1. Consider the 2-user Gaussian interference channel where the desired user employs $M_{1}$-PAM and the interferer uses $M_{2}$-PAM. For the interference-ignorant (II) detector, there exists a threshold $\mathrm{SIR}_{t h, I I}$, given by

$$
\operatorname{SIR}_{t h, I I}=\left(M_{1}+1\right)\left(M_{1}-1\right)\left(M_{2}-1\right) /\left(M_{2}+1\right),
$$

such that, when $\mathrm{SIR} \leq \mathrm{SIR}_{t h, I I}$, the symbol error rate of the interference-ignorant detector reaches an error floor, i.e.,

$$
\mathrm{P}_{e, I I} \rightarrow \mathrm{P}_{e, \text { floor }, I I} \neq 0 \text { as } \mathrm{SNR} \rightarrow \infty .
$$

For the SIC detector, there exist threshold values $\mathrm{SIR}_{t h, S I C, 1}$ and $\mathrm{SIR}_{t h, S I C, 2}$ given by

$\operatorname{SIR}_{t h, S I C, 1}=\left(M_{1}+1\right) /\left\{\left(M_{1}-1\right)\left(M_{2}-1\right)\left(M_{2}+1\right)\right\}$, and $\operatorname{SIR}_{t h, S I C, 2}=4\left(M_{1}+1\right)\left(M_{1}-1\right)\left(M_{2}-1\right) /\left(M_{2}+1\right)$, 
TABLE I

SIR THRESHOLD VALUES IN DB

\begin{tabular}{|c|c|c|c|}
\hline$\left(M_{1}, M_{2}\right)$ & $\mathrm{SIR}_{t h, I I}$ & $\mathrm{SIR}_{t h, S I C, 1}$ & $\mathrm{SIR}_{t h, S I C, 2}$ \\
\hline$(2,2)$ & 0 & 0 & 6.02 \\
\hline$(2,4)$ & 2.55 & -6.99 & 8.57 \\
\hline$(2,8)$ & 3.68 & -13.22 & 9.70 \\
\hline$(4,2)$ & 6.99 & -2.55 & 13.01 \\
\hline$(4,4)$ & 9.54 & -9.54 & 15.56 \\
\hline$(4,8)$ & 10.67 & -15.77 & 16.69 \\
\hline$(8,2)$ & 13.22 & -3.68 & 19.24 \\
\hline$(8,4)$ & 15.77 & -10.67 & 21.80 \\
\hline$(8,8)$ & 16.90 & -16.90 & 22.92 \\
\hline
\end{tabular}

such that, when $\operatorname{SIR}_{t h, S I C, 1} \leq \mathrm{SIR} \leq \mathrm{SIR}_{t h, S I C, 2}$, the symbol error rate of the SIC detector reaches an error floor:

$$
\mathrm{P}_{e, S I C} \rightarrow \mathrm{P}_{e, \text { floor }, \text { SIC }} \neq 0 \text { as } \mathrm{SNR} \rightarrow \infty .
$$

The optimal ML and joint MD detectors do not exhibit error floors, except for a finite number of SIR values at which the received constellation points for different values of the desired signals are colocated.

It can be easily shown that the threshold values satisfy the following relationship for $M_{1} \geq 2$ and $M_{2} \geq 2$ :

$$
\mathrm{SIR}_{t h, S I C, 1} \leq \operatorname{SIR}_{t h, I I}<\operatorname{SIR}_{t h, S I C, 2}=4 \operatorname{SIR}_{t h, I I} .
$$

Thus, both the interference-ignorant detector and the SIC detector have an error floor for $\mathrm{SIR}_{t h, S I C, 1} \leq \mathrm{SIR} \leq \mathrm{SIR}_{t h, I I}$. Table I shows some sample SIR threshold values. As can be seen in the table, the gap between $\operatorname{SIR}_{t h, S I C, 1}$ and $\operatorname{SIR}_{t h, I I}$ increases as $\left(M_{1}, M_{2}\right)$ increases. Thus, for larger constellation size, the optimal ML or the joint MD detectors should be employed instead of the existing detectors.

The results above shed light on the structure of optimal detection in the interference channel. On one hand, the fact that the joint detector outperforms the interference-ignorant detector implies that detecting interference is beneficial even when the interference signal is not of interest. On the other hand, the fact that the joint detector outperforms the SIC detector suggests that successive cancellation is too aggressive in relying on the successful detection of the entire interference signal-hard decisions on the interfering signal can lead to error propagation, which is detrimental to the overall performance. Instead, joint detection should be applied, which allows "soft" decoding of the interference signal to achieve the optimal performance.

\section{Complexity}

The interference-ignorant, the SIC, and the joint MD detector can be implemented by comparing the received symbol with the locations of the boundaries of the decision regions. Thus, the number of comparisons that are required serve as a good measure for the complexity. The interference-ignorant detector compares the received signal with the $M_{1}-1$ decision boundaries between the constellation points of the desired signal. Thus, the interference-ignorant detector requires at most $M_{1}-1$ comparisons. The SIC detector needs at most
$M_{2}-1$ comparisons for the detection of the interference and at most $M_{1}-1$ comparisons for the detection of the desired signal. Thus, the SIC detector requires at most $M_{1}+M_{2}-2$ comparisons. The joint MD detector needs to compare the received signal with the decision regions of $M_{1} M_{2}$ points of the combined constellation. Thus, the maximum number of comparisons for the minimum-distance detector is equal to $M_{1} M_{2}-1$. Although more comparisons are needed by the joint MD detector as compared to its interference-ignorant and SIC counterparts, this increase in complexity is relatively mild when compared to other parts of the receiver (such as a Viterbi decoder which may follow the detector). On the other hand, the optimal ML detector requires the calculation of all $M_{1} M_{2}$ Euclidean distances between the received symbol and the combined constellation points, which is significantly more complex than the other detectors.

\section{Simulation Results}

The performance of the various detectors is evaluated by Monte Carlo simulation to corroborate the theoretical results. Fig. 2 shows the SER for the interference-ignorant detector, the SIC detector, and the joint MD detector when 2-PAM is used by both transmitters for both the strong interference case of $\mathrm{SIR}=-3 \mathrm{~dB}$ and the weak interference case of $\mathrm{SIR}=3 \mathrm{~dB}$. It can be seen that, when the interference is strong, both the MD and SIC detectors perform well, while the interference-ignorant detector performs poorly. When the interference is weak, both the MD and interference-ignorant detectors perform well, while SIC performs poorly, as one would expect. In fact, the joint $\mathrm{MD}$ detector performs better for $\mathrm{SIR}=-3 \mathrm{~dB}$ than for $\mathrm{SIR}=3 \mathrm{~dB}$, which agrees with information theoretic results for the strong interference channel. The figure also contains theoretical exact SER curves that agree very well with the simulation results.

In Fig. 3, the joint MD detector and the optimal ML detector are compared. It can be seen that the lower-complexity MD detector matches the performance of the optimal ML detector almost completely, except at very low SNRs. Thus, MD is a viable alternative to ML.

Fig. 4 shows the SER performance when 4-PAM is used at both transmitters. A low interference case $(\mathrm{SIR}=3 \mathrm{~dB})$ and a high interference case $(\mathrm{SIR}=-3 \mathrm{~dB})$ are considered. In both cases, the MD and ML detectors perform well, while both the interference-ignorant and the SIC detectors show poor performance. This agrees with Theorem 1 , as $\operatorname{SIR}_{t h, I I}=9.54 \mathrm{~dB}$, $\mathrm{SIR}_{t h, S I C, 1}=-9.54 \mathrm{~dB}$ and $\mathrm{SIR}_{t h, S I C, 2}=15.56 \mathrm{~dB}$ for 4-PAM constellations. Therefore, SIR $= \pm 3 \mathrm{~dB}$ falls in an intermediate range where both conventional detectors fail to perform satisfactorily. This illustrates that the use of the joint MD or ML detectors is crucial for enabling 4-PAM signaling in this SIR range over the interference channel, which would otherwise be impossible.

Finally, Fig. 5 shows the SER when 4-QAM is used by both transmitters over a Rayleigh fading channel with i.i.d. complex Gaussian channel gains and uniform phase. The interferenceignorant detector and the SIC detector suffer significantly from 


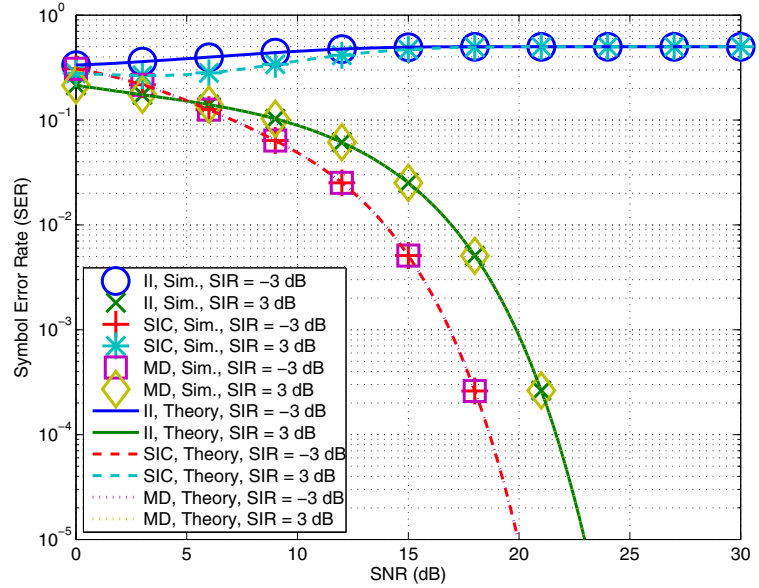

Fig. 2. SER for the interference-ignorant (II) detector, the SIC detector, and the joint MD detector when 2-PAM is used by both transmitters.

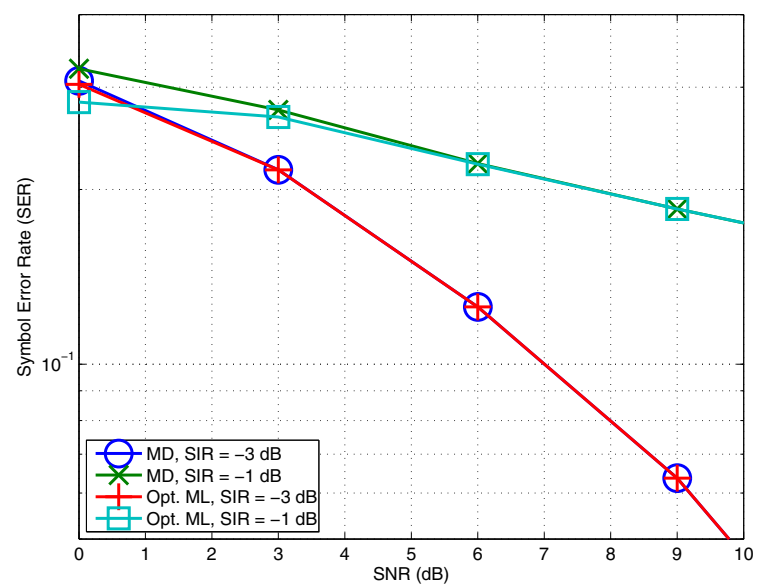

Fig. 3. SER for the joint MD detector and the optimal ML detector when 2-PAM is used by both transmitters.

fading at an average SIR of $6 \mathrm{~dB}$, showing an error floor The ordered SIC, which switches between the conventional detector and the SIC detector (depending on the instantaneous SIR with respect to $0 \mathrm{~dB}$ ), has slightly better performance, but still suffers from an error floor. On the other hand, the joint MD and the optimal ML detector do not exhibit an error floor, thus illustrating the value of joint detection.

\section{CONCLUSION}

This paper derives the joint MD detector and the optimal ML detector for the Gaussian interference channel when the transmitters employ finite constellations. The proposed detectors exploit the discrete nature of the modulation format. It is shown by analysis and by simulation that the proposed detectors outperform the interference-ignorant and SIC detectors for high-order modulation schemes and are not subject to error floors. Thus, the optimal detector for the Gaussian interference channel should neither completely ignore nor completely decode the interference-it should do something in-between.

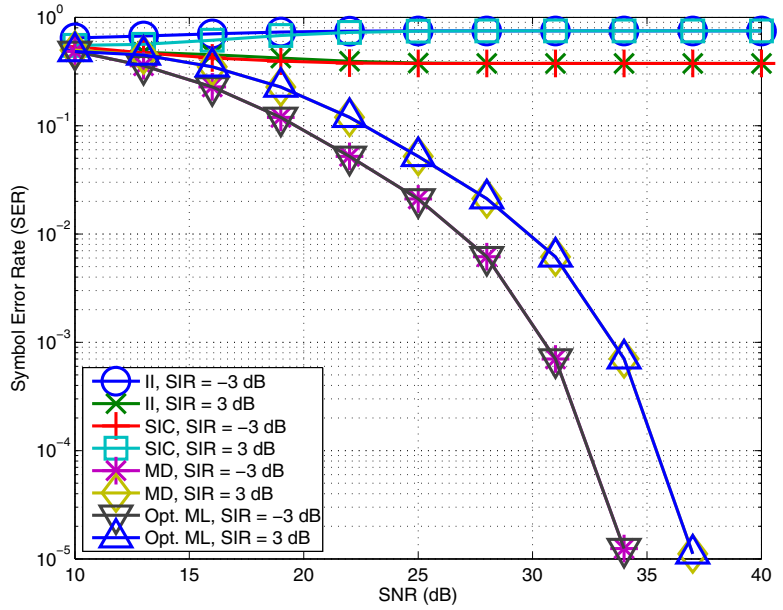

Fig. 4. SER when 4-PAM is used by both transmitters.

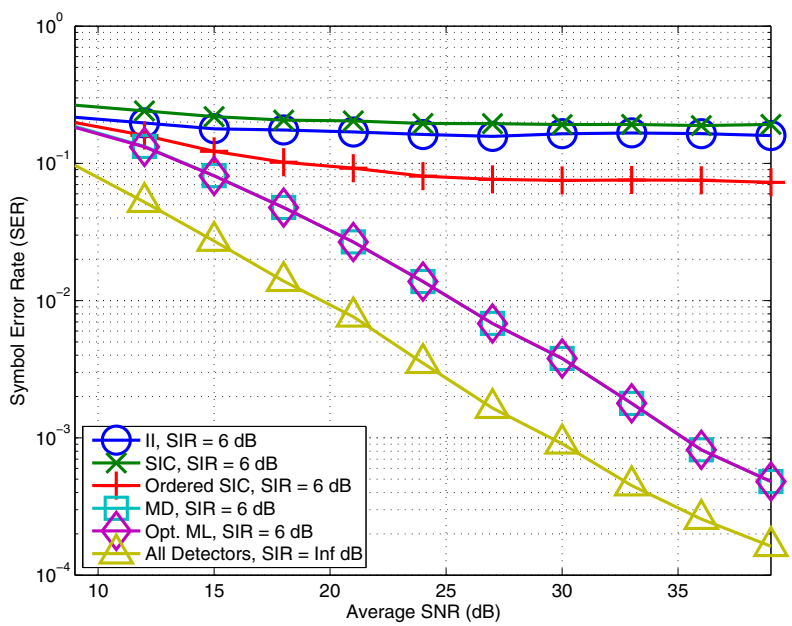

Fig. 5. SER in a Rayleigh fading channel when 4-QAM is used by both transmitters.

\section{REFERENCES}

[1] J. Lee, R. V. Sonalkar, and J. M. Cioffi, "Multi-user discrete bit-loading for DMT-based DSL systems," in Proc. IEEE Global Telecommun. Conf. (GLOBECOM), vol. 2, pp. 1259-1263, 2002.

[2] W. Yu, G. Ginis, and J. M. Cioffi, "Distributed multiuser power control for digital subscriber lines," IEEE J. Select. Areas Commun., vol. 20, pp. 1105-1115, June 2002.

[3] R. Cendrillon, W. Yu, M. Moonen, J. Verlinder, and T. Bostoen, "Optimal multi-user spectrum management for digital subscriber lines," IEEE Trans. Commun., vol. 54, pp. 922-933, May 2006.

[4] A. B. Carleial, "A case where interference does not reduce capacity," IEEE Trans. Inform. Theory, vol. IT-21, pp. 569-570, Sep. 1975.

[5] T. S. Han and K. Kobayashi, "A new achievable rate region for the interference channel," IEEE Trans. Inf. Theory, vol. IT-27, pp. 49-60, Jan. 1981.

[6] R. H. Etkin, D. Tse, and H. Wang, "Gaussian interference channel capacity to within one bit," IEEE Trans. Inf. Theory, vol. 54, no. 12, pp. 5534-5562, Dec. 2008.

[7] S. Verdu, Multiuser Detection, Cambridge Univ. Press, 1998.

[8] J. M. Cioffi, Course notes for Digital Communication: Signal Processing. Available at http://www.stanford.edu/group/cioffi/.

[9] J. Lee and D. Toumpakaris, "Optimal Symbol Detection in the Presence of Non-Gaussian Interference," submitted to IEEE Trans. Commun., available at http://www.wltl.ee.upatras.gr/drafts/detectorsIC.pdf. 